\title{
A REVIEW - SOLUBILITY ENHANCEMENT OF POORLY WATER SOLUBLE DRUGS
}

\author{
M. S. Patil ${ }^{* 1}$, A. A. Shirkhedkar ${ }^{2}$ \\ ${ }^{1}$ Shri. D.D.Vispute College of Pharmacy \& Research Center, Devad-Vichumbe, Panvel, Raigad, 410206. \\ ${ }^{2}$ R.C.Patel Institute of Pharmaceutical Education \& Research, Shirpur, Dhule, 425405. \\ *Corresponding author e-mail: mukeshpharma7@gmail.com
}

Received: 08 May 2016

Revised: 14 June 2016

Accepted: 22 June 2016

\begin{abstract}
:
Improvement in solubility of drug is a very formidable task in drug development. Approximately $40 \%$ of the new chemical entities currently being discovered are poorly water soluble drugs. The solubility and dissolution properties of drugs play a pivotal role in the process of formulation development. Among all newly discovered chemical entities most of the drugs are lipophilic and fail to reach market due to their poor water solubility. The ability to deliver poorly soluble drugs will grow in significance in the coming years. Similarly, generic drug manufacturers will need to exercise economically efficient methods of delivery as more low solubility drugs go off patent, in order to maintain a competitive edge and sufficiently compete as profit margins shrink in this price-sensitive industry. For this reason solubility enhancement is an important in future.
\end{abstract}

Key words: Dissolution, Lipophilic, Poor solubility.

\section{INTRODUCTION:}

It is well known that the therapeutic effectiveness of a drug depends upon the solubility of drug molecules. Solubility is one of the important parameter to achieve desired concentration of drug in systemic circulation for pharmacological response to be shown[1]. More than $40 \%$ of new candidates entering drug development pipeline fail because of non-optimal biopharmaceutical properties. Over the past few years, tools of drug discovery have caused an appreciable shift in biopharmaceutical properties ${ }^{[2]}$. Poorly water-soluble drugs involve many difficulties in the development of pharmaceutical dosage forms for oral delivery systems due to their low bio-availability ${ }^{[3]}$. Almost more than $90 \%$ drugs are orally administered. Drug absorption sufficient and reproducible bioavaiblity, pharmacokinetic profile of orally administered drug substances is highly dependent on solubility of that compound in aqueous medium[4].

\section{SOLUBILITY}

The term solubility is the property of a solid, liquid, or gaseous chemical substances called solute to dissolve in a solid, liquid, or gaseous solvent to form a homogeneous solution of the maximum quantity of solute in a certain quantity of solvent at specified temperature and pressure ${ }^{[5]}$.

\section{NEED OF SOLUBILITY ENHANCEMENT}

Drug absorption from the gastrointestinal tract can be limited by a variety of factors, most significant contributors being poor aqueous solubility and poor membrane permeability of the drug molecule. When delivering an active agent orally it must first 
dissolve in gastric and/or intestinal fluids before it can permeate the membranes of the GI tract to reach systemic circulation. Therefore, two areas of pharmaceutical research that focus on enhancing the oral bioavailability of active agents include; enhancing solubility and dissolution rate of poorly water-soluble drugs and enhancing permeability of poorly water soluble drugs. The Biopharmaceutical Classification system (BCS) is a scientific structure for classifying a drug substance based on its aqueous solubility and intestinal permeability[6]. The terminology of solubility is depicted in table 1.

Table 1: Terminology for solubility

\begin{tabular}{cc}
\hline Terminology & $\begin{array}{c}\text { Parts of solvent required for one } \\
\text { part of solute }\end{array}$ \\
\hline Very Soluble & $<1$ \\
Freely soluble & $1-10$ \\
Soluble & $10-30$ \\
Sparingly soluble & $30-100$ \\
Slightly & $100-1000$ \\
Very slightly soluble & $1000-10,000$ \\
Insoluble & $>10,000$ \\
\hline
\end{tabular}

\section{FACTORS AFFECTING THE SOLUBILITY}

There are mainly four factors which affects the solubility of the drugs; they are

1) Solute- solvent relationship,

2) Temperature,

3) Particle size and

4) Molecular size.

\section{Solute-Solvent Relations}

The relation between the solute and solvent is crucial in predicting solubility. Strong solute-solvent attractions are associated to greater solubility while weak solute-solvent attractions are associated to lesser solubility. The nature of solute and solvent based on concentration of solute in specific quantity of solvent at specific temperature. Successively, polar solutes tend to dissolve best in polar solvents whereas non-polar solutes incline to dissolve best in non-polar solvents. In the case of a polar solute and non-polar solvent www.ijapbjournal.com (or vice versa), it lead to be insoluble or only soluble to a miniscule degree. The general rule to understand is "Like Dissolves Like" [7].

\section{Temperature}

There is major influence of temperature on the solubility of solute. If the solution process absorbs energy then the solubility will enhance with rising temperature. If the solution process discharges energy then the solubility will diminish with increasing temperature ${ }^{[8]}$.

\section{Particle size}

Particle size can also affect solubility. As particle size reduced, the surface area to volume ratio increases. As the surface area of particle increases then it causes better interaction with solvent ${ }^{[9]}$.

\section{Molecular size}

Solubility is affected by molecular size of particle. The solubility of the substance is reduced when molecules have higher 
molecular weight and higher molecular size because larger molecules are more difficult to surround with solvent molecules in order to solvate the substance.

\section{TECHNIQUES FOR SOLUBILITY ENHANCEMENT}

\section{Particle size reduction:}

The solubility of drug is often intrinsically related to drug particle size as a particle becomes smaller, the surface area to volume ratio increases. The larger surface area allows a greater interaction with the solvent which cause increase in solubility. Conventional methods of particle size reduction, such as comminution and spray drying, rely upon mechanical stress to disaggregate the active compound[10].

\section{Complexation}

a. Physical Mixture: Active drug with suitable polymer in different ratios mixed in a mortar for about one hour with constant trituration. The mixture is passed through sieve no. 80 and stored in dessicator over fused calcium chloride.

b. Kneading method: Active drug with suitable polymer in different ratios is added to the mortar and triturated with small quantity of ethanol to prepare slurry. Slowly the drug is incorporated into the slurry with constant trituration. The prepared slurry is then air dried at $25^{\circ} \mathrm{C}$ for $24 \mathrm{hrs}$. The resultant product is pulverised and passed through sieve no. 80 and stored in dessicator over fused calcium chloride[11].

c. Co-precipitate method: Active drug is dissolved in ethanol at room temprature and suitable polymer is dissolved in distilled water. Different molar ratios of active drug and suitable polymers are mixed respectively. The mixture is stirred at room temprature for one hour and the solvent is evaporated. The resultant mass is pulverised and passed www.ijapbjournal.com through sieve no. 80 and stored in a desiccator[12].

\section{Hydrotropy}

Hydrotropy is a solubilization phenomenon whereby addition of large amount of a second solute results in an increase in the aqueous solubility of another solute. Concentrated aqueous hydrotropic solutions of sodium benzoate, sodium salicylate, urea, nicotinamide, sodium citrate and sodium acetate have been observed to enhance the aqueous solubilities of many poorly watersoluble drugs ${ }^{[13-14]}$. The term Hydrotropy was coined by Carl Neuberg in 1916 but the practical implications were introduced as late as 1976 by Thoma and coworkers[15]

Mechanism of action of Hydrotropes: Hydrotropes are the compounds having both an anionic group and a hydrophobic aromatic ring or ring system. The hydrophilicity increase by anionic group and the ring system interacts with the solute to be dissolved[16]. The mechanism involved in hydrotropy is related to complexation which involves interaction between lipophilic drugs and the hydrotropic agents such as urea, nicotinamide, sodium alginate, sodium benzoate etc ${ }^{[17]}$. The classification of hydrotorpes is shown in table 2.

\section{Advantages of hydrotropy method:}

1. In the hydrotropy method solvent character is independent of $\mathrm{pH}$, has high selectivity and does not require emulsification.

2. In this method simply mix the drug with the hydrotropes in water.

3. It does not require chemical modification of hydrophobic drugs, use of organic solvents or preparation of emulsion system. 


\section{Mixed Hydrotropy}

In mixed hydrotropy method the blends of hydrotropes are used. In blends of hydrotropes, the combination of hydrotropes gives synergistic effect on solubility of poorly water soluble drug. By reducing the concentration of individual hydrotropic agent, we reduce the side effect of hydrotropes. It is new, simple, cost-effective, safe, accurate, precise and environmental friendly method for the analysis (titrimetric and spectrophotometric) of poorly watersoluble drugs titrimetric and spectrophotometric precluding the use of organic solvents. As example, in case of ketoprofen by using $1.25 \mathrm{M}$ sodium citrate increase the solubility to 180 fold as compared to the solubility in distilled water. Maheshwari and co-workers increased solubility of paracetamol using urea and of aceclofenac using mixed hydrotropic phenomenon using urea and sodium acetate[19].

\section{Advantages of mixed hydrotropy} method[20]

1. It may reduce the large total concentration of hydrotropic agents necessary to bring about modest increase in solubility by employing combination of agents in lower concentration.
2. The use of hydrotropic solubilizers as permeation enhancers.

3. Application of mixed- hydrotropy to develop injection dosage forms of poorly water soluble drugs.

4. Application of hydrotropic solubilisation in nanotechnology (by controlled precipitation).

5. Application of hydrotropic solubilisation in extraction of active constituents from crude drugs.

\section{Application of hydrotropy in pharmacy:}

1. Preparation of dry syrups (for reconstitution) of poorly water-soluble drugs.

2. Quantitative estimations of poorly water soluble drugs by UV-Visible spectrophotometric analysis precluding the use of organic solvents.

3. Quantitative estimations of poorly water soluble drugs by titrimetric analysis. Such as ibuprofen, flurbiprofen.

The solubility enhancement of poorly water soluble drug using different hydrotropes is shown in table 3.

Table 2: Classification of Hydrotropes ${ }^{[18]}$

\begin{tabular}{cc}
\hline Class of Hydrotropes & Example \\
\hline Aromatic Anionics & $\begin{array}{c}\text { Sodium Benzoate, Sodium Salicylate, Sodium } \\
\text { Benzene sulphonate, Sodium cinnamate. }\end{array}$ \\
Aromatic Cationics & $\begin{array}{c}\text { Para amino benzoic acid hydrochloride, } \\
\text { Procaine Hydrochloride, Caffeine }\end{array}$ \\
Aliphatic \& Linear Anionics & Sodium Alkanote \\
\hline
\end{tabular}


Table 3: Solubility enhancement of poorly water soluble drug by using Hydrotropes

\begin{tabular}{|c|c|c|c|}
\hline S. No. & Drug & Hydrotropic Agent & Increase in Solubility \\
\hline 1. & Hydrochlorothiazide ${ }^{[21]}$ & Nicotinamide & 43 times \\
\hline 2. & Salbutamol sulphate[22] & Nicotinamide & 17 times \\
\hline 3. & Cefixime ${ }^{[23]}$ & $\begin{array}{l}\text { Ammonium acetate, Pot. Acetate, } \\
\text { Potassium Citrate, Sodium Citrate, } \\
\text { Urea }\end{array}$ & 10 times \\
\hline 4. & Ketoprofen ${ }^{24]}$ & Pot. Acetate & 210 times \\
\hline 5. & Lovastatin [25] & Sodium Acetate & 6 times \\
\hline 6. & Ornidazole ${ }^{[26]}$ & Ibuprofen sodium & 8 times \\
\hline 7. & Amlodipine besylate[27] & Sodium Acetate & 75 times \\
\hline 8. & Atenolol[28] & Metformine $\mathrm{HCl}$ & 3 times \\
\hline 9. & Theophylline[29] & Sodium Salicylate & 400 times \\
\hline 10. & $\begin{array}{l}\text { Eprosartan Mesylate } \\
\text { Hydrochlorothiazide }^{[30]}\end{array}$ & $\begin{array}{l}\text { Sodium Acetate, } \\
\text { Urea }\end{array}$ & $\begin{array}{l}56 \text { times } \\
74 \text { times }\end{array}$ \\
\hline 11. & Diclofenac Sodium[31] & Urea, Sodium Citrate & 250 times \\
\hline 12. & Albendazole[32] & Nicotinamide & 17 times \\
\hline 13. & Ibuprofen ${ }^{[33]}$ & Sodium Benzoate & 100 times \\
\hline 14. & Thioquanine[34] & Nicotinamide & 10 times \\
\hline 15. & Rapamycine[35] & $\begin{array}{l}\text { Benzyl alcohol, Benzyl benzoate, } \\
\text { Benzoic Acid }\end{array}$ & 1000 times \\
\hline 16. & Riboflavin ${ }^{[36]}$ & Caffeine & 5 times \\
\hline 17. & Carbamazepine ${ }^{[37]}$ & Nicotinamide & 2.5 times \\
\hline 18. & Halofantrine ${ }^{38]}$ & Caffeine, Nicotinamide & 100 times \\
\hline 19. & Nifedipine ${ }^{[39]}$ & 4-Sulphonic calyx[8] arene & 3 times \\
\hline 20. & Rafecoxib ${ }^{40]}$ & Urea, Nicotinamide & 5 times \\
\hline 21. & Aceclofenac ${ }^{411]}$ & Sodium Salicylate & 400 times \\
\hline 22. & Diacerein $^{[42]}$ & Urea, Pot Citrate & 150 times \\
\hline 23. & Frusemide[43] & $\begin{array}{l}\text { Urea, Sodium Acetate, Sodium } \\
\text { Citrate }\end{array}$ & 15 times \\
\hline 24. & Aspirin ${ }^{44]}$ & Ibuprofen sodium & 5 times \\
\hline 25. & Atorvastatin[45] & Urea & 6-7 times \\
\hline 26. & Famotidine ${ }^{46]}$ & Sodium Salicylate & 25 fold \\
\hline 27. & Cefadroxil[47] & Urea & 10 times \\
\hline 28. & Naproxen[48] & Ibuprofen sodium & 350 times \\
\hline 29. & Gatifloxacin $^{[49]}$ & N,N dimethyl urea, Sodi. Citrate & 15 times \\
\hline 30. & Lamotrigine[50] & $\begin{array}{l}\text { Urea, Sodium Benzoate, } \\
\text { Nicotinamide }\end{array}$ & 15 times \\
\hline
\end{tabular}


31. Glipizide ${ }^{51]}$

32. Pacilitaxel[52]

33. Amlodipine[53]

34. Chartreusin[54]
Sodium Benzoate, Sodium Acetate, Sodium Salicylate

Nicotinamide

Urea

Sodium Benzoate
6 times

25 fold

10 times

20 times

\section{Solid dispersion Techniques}

The term solid dispersion refers to the dispersion of one or more active ingredients in an inert carrier or matrix at solid state prepared by the melting (fusion), "solvent or the melting-solvent method". The dispersion of a drug or drugs in a solid diluent or diluents by traditional mechanical mixing is not included in this category. The solid dispersion may also be called solid-state dispersion[55].

a. The fusion (melt) method: Accurately weighed amounts of carrier(s) are placed in an aluminum pan on a hot plate and melted, with constant stirring, at a temperature of about $60^{\circ} \mathrm{C}$. An accurately weighed amount of active drug is incorporated into the melted carrier(s) with stirring to ensure homogeneity. The mixture is heated until a clear homogeneous melt is obtained. The pan is then removed from the hot plate and allowed to cool at room temperature.

b. The solvent method: Accurately weighed amounts of active drug and carrier(s) are dissolved in minimum quantities of chloroform in a round-bottom flask. The solvent is removed using a rotary evaporator. The resultant solid dispersion is transferred to an aluminum pan and allowed to dry at room temperature[56].

c. Dropping method: A solid dispersion of a melted drug-carrier mixture is measured and then dropped onto a plate where, it solidifies into round particles. The size and shape of the particles can be influenced by factors such as the viscosity of the melt and the size of the www.ijapbjournal.com pipette. Because viscosity is highly temperature dependent, it is very important to adjust the temperature so that when the melt is dropped onto the plate it solidifies to a spherical shape[13].

\section{Spray drying techniques}

a. Preparation of microparticles by spray drying: Spray dried particles consisting of active drug only and drug/suitable polymer in different ratios are prepared by dissolving the drug or drug/polymer mixture in ethanol/water solution. The solution is spray dried using mini spray dryer. The formed microparticles are separated using cyclone separator, collected and stored in a desiccator at ambient temperature until ready to be used.

\section{b. Preparation of microparticles by spray} chilling: Spray chilled particles are prepared by melting the drug or drug/suitable polymer mixture in different ratios at $90^{\circ} \mathrm{C}$. The melt is kept at $90^{\circ} \mathrm{C}$ and atomised with a specially constructed pneumatic nozzle into air kept at $20^{\circ} \mathrm{C}$. The particles are collected using cyclone separator and stored in a desiccator[14].

\section{Supercritical Fluid Technologies}

a. Supercritical anti-solvent (SAS) precipitation: The SAS apparatus works in a continuous co-current mode and it consists of a precipitator in which the antisolvent and the liquid solution are separately fed to the top of the chamber and are continuously discharged from the bottom. The liquid solution is pumped into the chamber by a 
high pressure piston pump. The antisolvent is delivered by means of a high pressure piston pump. The precipitator is a cylindrical vessel with an inner volume of $500 \mathrm{~cm}^{3}$. The liquid solution is delivered into the chamber through a stainless steel nozzle. The supercritical carbon dioxide is heated before entering the precipitator in a tube section by an electric cable, which is connected to a temperature controller. The precipitator is heated by means of two electric thin bands heater also connected to a temperature controller. A filter of sintered steel with sufficient porosity is placed at the bottom of the vessel to collect the particles produced. The solvents are separated and recovered from a second vessel[56].

b. Gas anti-solvent recrystalllization: It is possible to induce rapid crystallisation by introducing the antisolvent gas into a solution containing dissolved solute. One of the requirements for this approach is that the carrier solvent and the supercritical fluid (SF) anti-solvent must be at least partially miscible.

\section{c. Solution-enhanced dispersion by} supercritical fluids: The drug solution and the SF are introduced simultaneously into the particle formation vessel using a co-axial nozzle arrangement causing rapid dispersion, mixing and extraction of the drug solution solvent by SF leading to very high supersaturation ratios. The temperature and pressure together with accurate metering of flow rates of drug solution and SF through a nozzle provide uniform conditions for particle formation. This helps to control the particle size of the product and by choosing an appropriate liquid solvent; it is possible to manipulate the particle morphology[57].

\section{Nanosuspensions Preparation Methods}

a. Media milling (Nanocrystal or Nanosystems): The nanosuspensions are prepared by using high-shear media mills. The milling chamber charged with milling media, water, drug and stabilizer is rotated at a very high shear rate under controlled temperatures for several days (at least 2-7 days). The high energy shear forces are generated as a result of the impaction of the milling media with the drug resulting into breaking of microparticulate drug to nanosized particles.

\section{b. Homogenization in water (Dissocubes):} Homogenization involves the forcing of the suspension under pressure through a valve having a narrow aperture. The instrument can be operated at pressure varying from 100 - 1500 bars (2800 -21300 psi) and up to 2000 bars with volume capacity of $40 \mathrm{ml}$.

\section{c. Combined precipitation and homogenization (Nanoedege): In this} technique, the precipitated suspension is homogenized leading to reduction in particle size and avoiding crystal growth.

d. Nanojet technology: This technique called opposite stream or nanojet technology, uses a chamber where a stream of suspension is divided into two or more parts, which colloid with each other at high pressure. The high shear force produced during the process results in particle size reduction.

\section{e. Emulsification-solvent evaporation} technique: This technique involves preparing a solution of drug followed by its emulsification in another liquid that is a nonsolvent for the drug. Evaporation of the solvent leads to precipitation of the drug. Crystal growth and particle aggregation can be controlled by creating high shear forces using a high-speed stirrer ${ }^{[58]}$. 


\section{Preparation of nanocrystals}

The preparation process involves two steps

a. Preparation of drug solution in organic solvents

Different concentrations of drug solution are prepared by preparing solution of drug in organic solvent (based on solubility of drug in particular solvent).

\section{b. Addition of drug solution in water}

Nanocrystals are prepared by adding the microliter quantity of drug solution to milliliter quantity of water quickly with continuous stirring on magnetic stirrer at $1000 \mathrm{rpm}$ solvent is removed by overnight stirring at $500 \mathrm{rpm}$. Then it is centrifuged at $5000 \mathrm{rpm}$ and the product is solidified[59].

\section{Nanopure XP technology}

PharmaSol used in its Nanopure XP technology, a pre-treatment step with subsequent homogenization to produce particles well below $100 \mathrm{~nm}$. Drug nanocrystals with a size of about $50 \mathrm{~nm}$ and below are distinctly smaller than the wavelength of the visible light, and so the nanosuspensions are translucent ${ }^{[60] .}$

\section{Co-solvent evaporation method}

The solvent evaporation of drug and polymer solution in different ratio is carried out by using a suitable evaporator. The solutions are prepared by dissolving drug in methanol and polymer in distilled water and mixing both solutions, which produces clear solution. The clear solution is evaporated in evaporator.

\section{Spray drying}

The solvent evaporation of drug and polymer solution in different ratios is carried out by using spray dryer. The solutions are prepared by dissolving drug in methanol and polymer in distilled water and mixing both solutions, which produces a clear solution. The solvent evaporated by using evaporator. The spray dried mixture of drug with polymer is obtained in 20-30 $\min ^{[61]}$.

\section{Formulation of self microemulsifying drug delivery systems}

A series of SMEDDS formulations are prepared using surfactant/cosurfactant combination and oil. Accurately weighed active drug is placed in a glass vial and oil, surfactant and cosurfactant are added. Then the components are mixed by gentle stirring and vortex mixing on a magnetic stirrer, until drug is perfectly dissolved. The mixture is stored at room temperature until further use[62].

\section{A chitosan-based solvent change approach}

Chitosan solution is prepared by soaking chitosan in 1\% glacial acetic acid for $3 \mathrm{~h}$. A weighed amount of the drug is dispersed in chitosan solution by using high dispersion homogenizer. This dispersion is then added to distilled water or sodium citrate solution to precipitate chitosan on drug crystals. The precipitate obtained is filtered through Whatmann No. 1 filter paper using vaccum filtration unit and dried. The dried product is then passed through sieve no. 60 to obtain a uniform size distribution[63].

\section{Preparation of dry elixir}

Dry elixir is prepared by a spray drying technique. A laboratory scale spray drying is carried out using the spray dryer with a standard nozzle. Different compositions of spraying solution are prepared. Drug is dissolved in ethanol, while dextrin and SLS are dissolved in distilled water. Each solution is pre-warmed to $55-60^{\circ} \mathrm{C}$ and then blended. SLS is employed to prevent spray- dried particles from attaching to the inner wall of spray-drying chamber, to produce freeflowing powder, to handle with easy and to 
increase the encapsulation of ethanol in the dry elixir. The final solution is delivered to spray dryer. The drug is collected in cyclone separator and stored in a conical tube[64].

\section{Preparation of drug composite particles}

Active drug is dissolved in methanol and the solution is then filtered through a nylon membrane to remove any particulate impurities. Next, polymers are dissolved in deionized water, which is used as an antisolvent. The drug solution is poured rapidly into the anti-solvent with magnetic stirring at a rate of $2500 \mathrm{rpm}$. After stirring, a suspension containing drug nanoparticles are obtained. This suspension is then processed via spray drying to generate drug composite particles. Spray drying is carried out using a laboratory scale spray dryer[65].

\section{Preparation of dihydrochloride salt form}

Active drug is suspended in $800 \mathrm{ml}$ of acetone and into the suspension heated under reflux, the anhydrous gas of hydrogen chloride is bubbled slowly. After about $30 \mathrm{~min}$, the suspension became a solution and in another 5-10 min, the precipitate of the salt is formed. The pass of hydrogen chloride lasted for $2 \mathrm{~h}$ and the mixture is allowed to stand overnight at room temperature. The product is collected by filtration, washed with acetone and dried at $105^{\circ} \mathrm{C}[66]$.

\section{Amorphous systems}

Amorphization is one of the techniques to enhance the solubility of poorly water soluble drugs ${ }^{[67]}$. Delivering the pharmaceutical active ingredient in the amorphous form is very attractive due to the potentially large increase in drug solubility, dissolution rate and bioavailability[68-69]. The amorphous form of drugs can have as much as a 10-1600 fold higher solubility than their crystalline forms ${ }^{[70]}$. The improvement in dissolution of amorphous systems can be attributed to improved wetting of the drug, deagglomeration and micellization of the drug with hydrophilic polymers and the high energy amorphous state of the drug[71]. However, the amorphous forms of drugs are physically unstable due to their higher energy state and may recrystallize over pharmaceutically relevant time scales, negating any solubility advantage[72]. The most typically used approach to stabilize an amorphous system is to combine it with pharmaceutically acceptable polymers, such as polyvinylprrolidone, polyvinylpyrrolidone vinyl acetate, polyethylene glycol and various hydroxypropylmethyl cellulose and polyacrylic acid derivatives [73-74]. Thermodynamically the drug has a lower chemical potential when mixed with a polymer, resulting in a change of crystallization driving force[75]. The long polymeric chains can sterically hinder the association between drug molecules and, thereby, inhibit the recrystallization of drug. In addition, the interaction between the drug and polymer provides an increased energy barrier for nucleation and, consequently, enhances the physical stability[76]. Amorphous drug-polymer systems are commonly characterized in terms of physical properties such as the glass transition temperature $(\mathrm{Tg})$, heat capacity and miscibility. Although it is still not completely clear as to how the polymer stabilizes the amorphous drug in the mixture, drug polymer miscibility is generally considered as one of the critical attributes that affect the stability of the amorphous systems, which in turn is dictated by the thermodynamics of mixing[77]. Amorphous systems are predominantly produced by solvent evaporation and melt extrusion methods [78-79]. 


\section{CONCLUSION}

Use of solubility characteristics in bioavailability, pharmaceutical actions and solubility enhancement of various poorly soluble compounds is a challenging task for researchers and pharmaceutical scientists. Dissolution enhancement of poorly water soluble drugs constitute an innovative approach, which overcome the problems of solubility and dissolution rate limiting step and provide a quick onset of action. Hydrotropic solubility enhancement is simple, cost effective, eco-friendly, and novel

\section{RERFERENCES:}

1. Mohanachandran PS, Sindhumol PG, Kiran TS. Enhancement of Solubility and Dissolution Rate: An Overview. Int. J. Comprehensive Pharm., 2010; 1(4): 1-10.

2. Thorat YS, Gonjari ID, Hosmani AH. Solubility Enhancement Techniques: A Review on Conventional and Novel Approaches. Int. J. Pharm. Sci. Res., 2011; 2(10): 2501-2513.

3. Chilukala S, Aparna C, Shastri N, Sadanandam M. Approaches To Enhance Solubility And Dissolution of Poorly Water Soluble Drug: Satranidazole. J. Pharm. Res., 2011; 4(11): 4135-4138.

4. Jatwani S, Rana AC, Singh G, Aggarwal G. An Overview on Solubility Enhancement Techniques for Poorly Soluble Drugs and Solid Dispersion as an Eminent Strategic Approac. Int. J. Pharm. Sci. Res., 2012; 3(4): 942-956.

5. Lachman L, Lieberman HA. The Theory and Practice of Industrial Pharmacy. $3^{\text {rd }}$ ed; CBS Publication \& Distributors Pvt. Ltd. 2009; 221.

6. Mofizur RM, Khalipha AB, Ahmed J, S. Haque. Methods of Solubility And Dissolution Enhancement For Poorly method. Previously nicotinamide, sodium benzoate, sodium acetate, urea have been utilised most frequently but nowadays novel hydrotropes such as metformin hydrochloride, ibuprofen sodium have been utilised, and many of their kind can be exploited as hydrotropes. Hydrotropy method haven't yet been commercialised as there are issues related to use of higher concentration of hydrotropic agent for formulation. But it can be used for analysis of APIs and finished dosage forms. It is a cost effective method because it does not contain organic solvents.

Water Soluble Drugs: A Review. World J. Pharm. Pharma. Sci. 2014, 3(5): 107-130.

7. Patil SK, Wagh KS, Parik VB, Akarte AM, Baviskar DT. Strategies for solubility enhancement of poorly soluble drug. Int. J. Pharm. Sci. Rev. Res., 2011; 8(2): 74-80.

8. Blagden N, Matas M, Gavan PT, York P. Crystal engineering of active pharmaceutical ingredients to improve solubility and dissolution rates. Adv. Drug Delivery Rev. 2007; 59(7): 617-630.

9. Chaudhari A, Nagachi U, Gulati N, Sharma VK, Khosa RK, Enhancement of solubilisation and bioavailability of poorly soluble drugs by physical and chemical modification; A recent review. J. Adv. Pharm. Edu. Res., 2012; 2(1): 32-67.

10. Gupta AK, Sehrawat SK. Bioavailability Enhancement of Poorly Water Soluble Drugs: A Review. Int. J. Pharm. Life Sci., 2011; 2(3): 640-650.

11. Pawar AR, Choudhari PD. Novel techniques for solubility, dissolution rate and bioavailability enhancement of class II \& IV drugs. Asian J. Biomed. Pharm. Sci., 2012; 13: 9-14. 
12. Das S, Pattanayak D, Sahu NP. Dissolution enhancement of nimesulide using HPßCD. J. Pharm. Res. 2012; 5(1): 508-510.

13. Shah SS, Pasha TY, Behera AK, Bhandari A. Solubility Enhancement and Physicochemical Characterization of Inclusion Complexes Of Itraconazole. Der Pharmacia Lettre. 2012; 4(1): 354-366.

14. Jain P., Goel A., Sharma S., Parmar M., Solubility Enhancement Techniques with Special Emphasis on Hydrotropy. Int. J of Pharma. Professional's Research. 2010; 1(1): 34-45.

15. Kapadiya N, Singhvi I, Mehta K, Karwani G, DhruboJS. Hydrotropy: a promising tool for enhancement of solubility: a review. Int. J. of Drug Devel. \& Research. 2011; 3(2): 26-33.

16. Zaheer A, Naveen M, Santosh MK, Imran K. Solubility enhancement of poorly water soluble drugs. Int. J. of Pharma tech, 2011; 3(1): 807-823.

17. Fribergand SE, Blute I. Liquid Detergents. Edited by Kuo-yann lai, CRC Press. 2005; 19-38.

18. Maheshwari RK, Dewangan, A, Soni, PK, Jain SK. Novel Application of Hydrotropic Solubilization in the Spectrophotometric Analysis of Paracetamol Tablet. Asian J. Chemistry. 2006; 18(4): 2879-2882.

19. Jain P., Goel A., Sharma S., Parmar M. Solubility Enhancement Techniques with Special Emphasis on Hydrotropy. Int. J. Pharm. Prof. Res., 2010; 1(1): 34-45.

20. Patel T, Patel LD, Patel T, Makwana S. Enhancement of dissolution of fenofibrate by solid dispersion technique, Int. J. Res. Pharm. Sci., 2010; 1(2): 127-132.

21. Maheshwari RK, Shukla RS. Novel method for spectrophotometric analysis of hydrochlorothiazide tablets using niacinamide as hydrotropic solubilizing agent. Asian J. Pharm., 2008; 2(1): 68-69.

22. Sundari N, Radhika T, Saranya V, Jayakumar C, Gandhi NN. Quantitative analysis of salbutamol bulk sample using nicotinamide hydrotrope, Int. J. Pharm. Pharma. Sci. Res. 2012; 2(1): 16-19.

23. Pareek V, Tambe SR, Bhalerao SB. Role of different hydrotropic agents in spectrophotometric and chromatographic estimation of cefixime, Int. J. Pharm. Bio. Sci., 2010; 1(3): 1-10.

24. Pandey S, Maheshwari RK. A novel spectrophotometric method for the estimation of ketoprofen in tablet dosage form using hydrotropic solubilisation phenomenon. World Appl. Sci. J. 201; 11(12): 1524-1527.

25. Patil DN. Spectroscopic determination of lovastatin by hydrotropic solubilization technique. Int. J. Pharm. Chem. Sci. 2012; 1(3): 1142-1144.

26. Maheshwari RK, Bishnoi SR, Kumar D, Krishna M. Quantitative spectrophotometric determination of ornidazole tablet formulations using ibuprofen sodium as hydrotropic solubilizing agent. Digest J. of Nanomaterials and Biostructures. 2010; 5(1): 97-100.

27. Jain N, Jain R, Jain A, Pandey SP, Jain DK. Spectrophotometric method development and validation for quantitative estimation of amlodipine besylate in bulk drug and their dosage forms by using hydrotropic agent. Eur. asian J. Anal. Chem. 2010; 5(3): 212-217.

28. Maheshwari RK, Agrawal A, Rathore A, Agrawal M. Eco-friendly spectrophotometic estimation of atenolol tablets using metformin hydrochloride as 
hydrotropic solubilizing agent. J. Global Pharm. Techn., 2010; 2(4): 93-96.

29. Jayakumar C, Deepak Kumar, Nesakumar D, Gandhi NN. Quantitative analysis of theophylline bulk sample using sodium salicylate hydrotrope, Int. J. Pharm. Sci., 2010; 2(4): 80-81.

30. Jain R, Sahu V, Jain N, Jain S. Mixed hydrotropy solubilization approach for quantitative estimation of eprosartan mesylate and hydrochlorthiazide by UV spectrophotometer, Pharm. Anal. Acta, 2011; 2(7): 1-4.

31. Gupta MM, Joshi VH, Amipara L, Patel V, Mahida M. Development and evaluation of diclofenac sodium solid dispersion by mixed hydrotropy. Int. J. Pharm. Res. Develop., 2011; 3(8): 90-96.

32. Balaji NJ, Kulkarni PK, Prabhuling VR, Hydrotropic solubilization of albendazole. Indian J. Pharm. Edu. Res. 2007; 41(2): 150-154.

33. Patel SK, Dinesh Kumar, Waghmode AP, Dhabale AS. Solubility enhancement of ibuprofen using hydrotropic agents. Int. J. Pharm. Life Sci., 2011; 2(2): 542-545.

34. Truelove J, Nassar RB, Chen NR, Hussain A, Solubility enhancement of some developmental anti-cancer nucleoside analogs by complexation with nicotinamide. Int. J. Pharm. 1984; 19; 1725.

35. Simamora P, Alvarez JM, Yalkowsky SH. Solubilization of rapamycin, Int. J. Pharm. 2001; 213: 25-29.

36. Cui Y. Parallel stacking of caffeine with riboflavin in aqueous solutions: The potential mechanism for hydrotropic solubilization of Riboflavin. Int. J. Pharm., 2010; 397; 36-43.
37. Shikhar A, Bommana MM, Gupta SS, Squillante E. Formulation development of carbamazepine-nicotinamide co-crystals complexed with $\gamma$-cyclodextrin using supercritical fluid process. J. Supercritical Fluids. 2011; 55; 1070-1078.

38. Lim LY, Go ML. Caffeine and nicotinamide enhances the aqueous solubility of the antimalarial agent halofantrine, Eur. J. Pharm. Sci., 2000; 10; 17-28.

39. Yang W, De Villiers MM. The solubilization of the poorly water soluble drug nifedipine by water soluble 4sulphonic calix[n]arenes. Eur. J. of Pharma. and Biopharma. 2004; 58; 629636.

40. Ahuja N, Katare OP, Singh B. Studies on dissolution enhancement and mathematical modelling of drug release of a poorly water-soluble drug using water-soluble carriers. Eur. J. Pharm. Biopharm., 2007; 65: 26-38.

41. Maheshwari RK, Moondra S. A novel method for quantitave determination of aceclofenac in Bulk drug and tablets using sodium salicylate as hydrotropic solubilising agent. J. Adv. Pharm. Tech. Res., 2010; 1(1): 78-82.

42. Pandey R, Patil PO, Patil MU, Deshmukh PK, Bari SB. Quantitave estimation of diacerein in bulk and in capsule formulation using hydrotropic solubilising agents by UVspectrophotometry and the first order derivative using the area under curve method. Pharm. Methods. 2012; 3(1): 4-8.

43. Maheshwari RK, Juneja C, Juneja N. Application of mixed-hydrotropic solubilization concept in spectrophotometric analysis of frusemide in tablet dosage form. The Pharma Res., 2010; 3: 243-248. 
44. Maheshwari RK, Saxena M, Gahlot $M$, Chaki R, Kinariwala M, Jagwani Y. Novel application of hydrotropic solubilizing additives in the estimation of aspirin in tablets. Ind. J. Pharm Sci., 2010; 72(5): 649-651.

45. Jadhav SD, Bhatia MS, Thamake SL, Pishawikar SA. Spectrophotometric methods for estimation of atorvastatin calcium form tablet dosage forms. Int. J. Pharm. Tech. Res., 2010; 2(3): 19481953.

46. Jayakumar C, Morais AB, Rajasekhar G, Reddy G, Gandhi NN. Quantitative analysis of famotidine bulk sample using sodium salicylate hydrotrope. Int. J. Inst. Pharm. Life Sci., 2012; 2(2): 98-103.

47. Shukla RS, Patel A, Soni ML, Modi V, Jaliwala YA. Quantitative spectrophotometric estimation of Cefadroxil using hydrotropic solubilization technique. Asian J. Pharm. 2008; 2: 146147.

48. Maheshwari RK, Wanare G, Chahar N, Joshi P, Nayak N. Quantitative estimation of naproxen in tablets using Ibuprofen sodium as hydrotropic agent. Indian J. Pharm. Sci. 2009; 71(3): 335-337.

49. Shrivastava R, Jain R, Patel S. Spectrophotometic analysis of gatifloxacin tablets using mixed hydrotropy. Int. J. Pharm. Sci. Res., 2011; 2(10): 2709-2711.

50. Patil AE. Spectrophotometric Estimation of lamotrigine in tablet dosage form by using mixed hydrotropy an eco-friendly method. Indo American J. Pharm. Res., 2013; 3(3): 2655-2661.

51. Shukla M, Rathore P, Jain A, Nayak S. Enhanced solubility study of glipizide using different solubilization techniques.
Int. J. Pharm. Pharma. Res., 2010; 2(2): 46-48.

52. Lee J, Lee SC, Acharya G, Chang C, Park K. Hydrotropic solubilization of pacilitaxel: of chemical structures for hydrotropic property. Pharm. Res., 2003; 20(7): 10221030.

53. Bernard S, Mathew M, Senthilkumar KL. Spectrophhotometric method of estimation of amlodipine besylate using hydrotropic solubilisation. J. Appl. Pharm. Sci., 2011; 1(9): 177 -180.

54. Poochikian GK, Cradock JC, Enhanced chartreusin solubility by hydroxybenzoate hydrotropy. J. Pharm. Res., 1979; 68(6): 2170-2171.

55. Joshi, V.B., Tejwani, R.W., Davidovich, M., Saharabudhe, V.P., Jemal, M., Bathala, M.S., Varia, S.A., Serajuddin, A.T.M. Bioavalability enhancement of a poorly water-soluble drug by solid dispersion in polyethylene glycol-polysobate80 mixture. 2004; 269: 251-258.

56. Amal A, Ebtessam AE. Dissolution of ibuprofen from spray dried and spray chilled particles. Pak. J. Pharm. Sci., 2010; 23(3): 284-290.

57. Ana RC, Duarte A, Gordillo MD, Margarida C. Preparation of ethyl cellulose/methyl cellulose blends by supercritical antisolvent precipitation. Int. J. Pharm., 2006; 311(1-2): 50-4.

58. Kakumanu V, Bansal AK. Supercritical fluid technology in pharmaceutical research. department of pharmaceutical technology (formulations). Business Briefing: Labtec. 2004; 70-72.

59. Kamble VA, Jagdale DM, Kadam VJ. Nanosuspension a novel drug delivery system. Int. J. Pharm. Bio Sci., 2010; 1(4): 352-360. 
60. Nanjwade BK. Derkar GK, Bechra HM, Nanjwade VK, Manvi FV. Design and characterization of nanocrystals of lovastatin for solubility and dissolution enhancement. Nanomedicine \& Nanotechnology. 2011; 2-7.

61. Yadav VB, Yadav AV. Enhancement of solubility and dissolution rate of bcs class ii pharmaceuticals by non aqueous granulation technique. Int. J. Pharm. Res. Dev., 2010; 1(12): 1-12.

62. Pandya P, Gattani S, Jain P, Khirwal L, Surana SJ. Co-solvent evaporation method for enhancement of solubility and dissolution rate of poorly aqueous soluble drug simvastatin: in vitro-in vivo evaluation. AAPS Pharmscitech. 2008; 9(4): 1247-1252.

63. Bora D, Borude P, Bhise K. Formulation and evaluation of self microemulsifying drug delivery systems of low solubility drug for enhanced solubility and dissolution. . Asian J. Biomed. Pharm. Sci. 2012; 2(15): 7-14.

64. Srinivas Mutalik, Parambil Anju A, Krishnan Manoja, Achutha Nayak Usha, Enhancement of Dissolution Rate and Bioavailability of Aceclofenac: A ChitosanBased Solvent Change Approach, Int. J. Pharm. 2008; 350, 279-290.

65. Seo-Ryung Kima, Jin-Ki Kima, Jeong-Sook Parkb, Chong-Kook Kimd. Dry elixir formulations of dexibuprofen for controlled release and enhanced oral bioavailability. Int. J. Pharm. 2011; 404: 301-307.

66. Zhanga Z, Yuan L, Wanga J, Hong Z, Jianfeng C. Irbesartan drug formulated as nanocomposite particles for the enhancement of the dissolution rate. Particuology. 2012; 10(4): 462-467.
67. Taoa T, Zhaoa Y, Zhoub B, Preparation and evaluation of itraconazole dihydrochloride for the solubility and dissolution rate enhancement. Int. J. Pharm. 2009; 367: 109-114.

68. Mohammad BJ, Valizadeh H, Mohammad $\mathrm{R}$, Shadbad S, Adibkia K. Cogrinding as an approach to enhance dissolution rate of a poorly water-soluble drug (gliclazide). Powder Tech., 2010; 197; 150-158.

69. Vervaet C, Baert L, Remon JP. Enhancement of in vitro drug release by using polyethylene glycol400 and PEG-40 hydrogenated castor oil in pellets made by extrusion/spheronisation. Int. J. Pharm., 1994; 108: 207-212.

70. Ito A, Watanabe T, Yada S, Hamaura T, Nakagami H, Higashi $\mathrm{K}$, Moribe $\mathrm{K}$, Yamamoto K. Prediction of recrystallization behavior of troglitazone/polyvinylpyrrolidone solid dispersion by solid-state NMR. Int. J. Pharm., 2010; 383(1-2): 8-23.

71. Hancock BC, Zografi G. Characteristics and significance of the amorphous state in pharmaceutical systems. J. Pharm. Sci., 1997; 86(1): 1-12.

72. Yu L. Amorphous pharmaceutical solids: preparation, characterization and stabilization. Adv. Drug Deliver Rev. 2001; 48(1): 27-42.

73. Hancock BC, Parks M. What is the true solubility advantage for amorphous pharmaceuticals. Pharm. Res., 2000; 17(4): 397-404.

74. Chokshi RJ, Zia H, Sandhu HK, Shah NH, Malick WA. Improving the dissolution rate of poorly water soluble drug by solid dispersion and solid solution: pros and cons. Drug Deliv., 2007; 14(1): 33-45. 
75. Konno H, Taylor LS. Influence of different polymers on the crystallization tendency of molecularly dispersed amorphous felodipine. J. Pharm. Sci., 2006; 95(12): 2692-2705.

76. Serajuddin AT. Solid dispersion of poorly water-soluble drugs: early promises, subsequent problems and recent breakthroughs. J. Pharm. Sci., 1999; 88(10): 1058-1066.

77. Ivanisevic I. Physical stability studies of miscible amorphous solid dispersions. J. Pharm. Sci., 2010; 99(9): 4005-4012.

78. Qian F, Huang J, Hussain MA. Drugpolymer solubility and miscibility: stability consideration and practical challenges in amorphous solid dispersion development. J. Pharm. Sci., 2010; 99(7): 2941-2947.

79. Yang J, Grey K, Doney J. An improved kinetics approach to describe the physical stability of amorphous solid dispersions. Int J. Pharm. 2010; 384(1-2): 24-31.

How to cite this article:

M. S. Patil, A. A. Shirkhedkar. A Review - Solubility Enhancement of Poorly Water Soluble Drugs. Int. J. Adv. Pharm. Biotech., 2016; 2(1): 10-24. 